\title{
Pioglitazone Loaded-PLGA-PEG Nanoparticles: Drug Release and Interactions
}

\author{
Marcelle Silva de Abreu, Maria Antonia Egea, Ana Cristina Calpena, Marta Espina, Maria Luisa García \\ Faculty of Pharmacy/University of Barcelona \\ Av Joan XII, s/n, Barcelona, Spain \\ marcelle.ievv@gmail.com; m.egea@ub.edu; anacalpena@ub.edu; m.espina@ub.edu; marisagarcia@ub.edu
}

\section{Extended Abstract \\ Introduction}

The main obstacle to transport drugs to the brain, for neurodegenerative diseases treatment, is the blood-brain barrier (BBB), acting as an immune and metabolic barrier [1]. Pioglitazone (PGZ) is an oral anti-diabetic from thiazolidinediones, agonist of the peroxisome proliferator-activated receptors (PPARs), which could play an important role on mechanisms of neurodegenerative diseases [2,3]. The main goal of this work was the PGZ association nanostructured systems, to nanoparticles (NPs) from poly (D,L-lactide-co-glycolide) poly(ethylene glycol) (PLGA-PEG) that are able to pass BBB.

\section{Materials and Methods}

PGZ-NPs were prepared by solvent displacement technique using polyvinyl alcohol as surfactant, PLGA-PEG, and acetone as organic solvent. The influence of several factors such as: $\mathrm{pH}$ of aqueous phase, and stabilizer and matrix concentration on physicochemical properties (particle size, polydispersity index [PI], zeta potential [ZP]) of NPs, a factorial design $2^{3}$ has been carried out. Morphology was analysed by transmission electron microscopy (TEM). Morphometry and ZP were determined by dynamic light scattering (DLS), and electrophoretic mobility, respectively. Drug loading efficiency was assessed by Ultra Performance Liquid Chromatography (UPLC). In vitro release studies of PGZ from NPs were performed Franz-type diffusion cells with dialysis cellulose membranes. Interactions drug-polymer have been studied by Differential Scanning Calorimetry and X-rays. Short-term stability was evaluated by optical analyzer Turbiscan ${ }^{\circledR}$ Lab (Formulaction).

\section{Results and Discussion}

Average size of PLGA-PEG-PGZ is suitable to pass BBB. IP decreases at low concentrations of PVA and PLGAPEG. The ZP, which is a measure of the surface charge of the particles, increases at low concentrations of PLGA-PEG and PVA. High association efficiency $(98.14 \%)$ was obtained. Morphology of the optimized particles was spherical with few aggregates being their average size similar to those obtained by DLS. Release profiles of PGZ from NPs, exhibit a quicker release at the beginning, due to the amount PGZ present on the surface of the NPs, followed by a slow release phase. From the values of Akaike's information (AIC) and coefficient of determination $\left(\mathrm{r}^{2}\right)$ (93.4 and 0.9535 respectively), the release curves of NPs and the free drug fit properly to the kinetics hyperbola model. Thermal methods and X-rays assay confirmed that the drug is dispersed inside NPs. Short-term stability NPs, analyzed through the backscattering profile of light, showed no significant changes compared to initial values.

\section{Conclusions}

In this work, PGZ loaded PLGA-PEG NPs has been developed by interfacial deposition procedure, with morphometric characteristics suitable to pass BBB.

\section{Acknowledgements}

Authors would like to thank to CAPES (Coordination for the Improvement of Higher Education Personnel) Brazil and the Spanish Ministry of Science and Innovation grant (MAT2014-59134R) for financial support. 


\section{References}

[1] C. Iadecola, "The overlap between neurodegenerative and vascular factors in the pathogenesis of dementia," Acta neuropathol., vol. 120, no. 3, pp. 287-296, 2010.

[2] J. Chen, S. Li, W. Sun, and J. Li, "Anti-diabetes drug pioglitazone ameliorates synaptic defects in ad transgenic mice by inhibiting cyclin-dependent kinase5 activity," Plos one, vol. 10, no. 4, pp. 1-12, 2015.

[3] T. Sato, H. Hanyu, K. Hirao, H. Kanetaka, H. Sakurai, and T. Iwamoto, "Efficacy of ppar-agonist pioglitazone in mild Alzheimer disease," Neurobiology of aging., vol. 32, no. 9, pp. 1626-1633, 2011. 